\title{
Fundamental Characteristics of Queues with Fluctuating Load
}

\begin{abstract}
Systems whose arrival or service rates fluctuate over time are very common, but are still not well understood analytically. Stationary formulas are poor predictors of systems with fluctuating load. When the arrival and service processes fluctuate in a Markovian manner, computational methods, such as Matrix-analytic and spectral analysis have been instrumental in the numerical evaluation of quantities like mean response time. However, such computational tools provide only limited insight into the functional behavior of the system with respect to its primitive input parameters: the arrival rates, service rates, and rate of fluctuation.

For example, the shape of the function that maps rate of fluctuation to mean response time is not well understood, even for an $M / M / 1$ system. Is this function increasing, decreasing, monotonic? How is its shape affected by the primitive input parameters? Is there a simple closed-form approximation for the shape of this curve? Turning to user experience: How is the performance experienced by a user arriving into a "high load" period different from that of a user arriving into a "low load" period, or simply a random user. Are there stochastic relations between these? In this paper, we provide the first answers to these very fundamental questions.
\end{abstract}

\section{INTRODUCTION}

\section{Motivation and model}

The vast majority of queueing models assume a stationary process in order to derive performance characteristics, such as mean response time or mean number in system. In reality, computer systems have arrival rates which fluctuate over time. Furthermore, when the arrival rate is high, it is common to try to compensate by increasing the service rate, possibly by adding additional servers.

System designers often try to use standard queueing theorems, such as the stationary $\mathrm{M} / \mathrm{M} / 1$ formulas, to predict the performance of their system. However, when the load fluctuates over time, it is not clear which stationary formula

Permission to make digital or hard copies of all or part of this work for personal or classroom use is granted without fee provided that copies are not made or distributed for profit or commercial advantage and that copies bear this notice and the full citation on the first page. To copy otherwise, to republish, to post on servers or to redistribute to lists, requires prior specific permission and/or a fee.

Copyright 200X ACM X-XXXXX-XX-X/XX/XX $\ldots \$ 5.00$. to use. One can try to average the load in some way over time, and use a stationary M/M/1 with the "average load," to predict system performance. However, as many system designers know, this is a very poor estimation of mean behavior. Furthermore, it completely ignores the differences in user perceived performance depending on whether the user arrives into a high-load or low-load state.

As people have become aware of the effects of fluctuating load, mathematical tools have been developed, such as matrix analytic methods and spectral analysis, which allow one to numerically evaluate systems in which the arrival rate and service rate change over time according to a Markovian process. While such tools provide numerical values for timeaverage behavior, they provide only limited insight into the functional behavior of the system with respect to the input parameters. These methods don't tell us how the mean response time is affected by the rate of fluctuation between high and low load, whether this is increasing, whether it is monotonic, etc. These methods don't give us a complete sense of how the results vary as a function of the other input primitives, such as the arrival rate and service rate, or which parameters are most important.

In order to consider such questions, we evaluate a specific model for fluctuating load, shown in Figure 1. The system alternates between a "high" state and a "low" state, according to a Markovian process, where the system is in "high" for an exponentially-distributed time with rate $\alpha^{H}$ and in the "low" state for an exponentially-distributed time with rate $\alpha^{L}$. While in the high state (respectively low state), arrivals occur according to a Poisson Process with rate $\lambda^{H}$ (respectively, $\lambda^{L}$ ). Also while in the high state (respectively low state), services complete with exponential rate $\mu_{H}$ (respectively, $\left.\mu^{L}\right)$. We define $\rho^{H}=\frac{\lambda^{H}}{\mu^{H}}$ and $\rho^{L}=\frac{\lambda^{L}}{\mu^{L}}$ and assume throughout that $\rho^{H} \geq \rho^{L}$ (specifically we do not assume any relationship between $\lambda^{H}$ and $\lambda^{L}$ or between $\mu^{H}$ and $\left.\mu^{L}\right)$. We allow $\rho^{H}>1$, provided that the system is still stable, as defined in Section 2. Note that the above model encompasses as special cases models with $\mathrm{ON} / \mathrm{OFF}$ arrival processes (where $\left.\lambda^{L}=0\right)$ and/or breakdowns $\left(\mu^{H}=0\right.$, in this case we define $\left.\rho^{H}=\infty\right)$. Even this simple Markovian model generates non-obvious and counter-intuitive behavior, and provides insight for more general models. In Section 3, we will also consider a more general variant of our model where we allow for a burst of arrivals at each arrival instant, where the burst size can have an arbitrary distribution. 


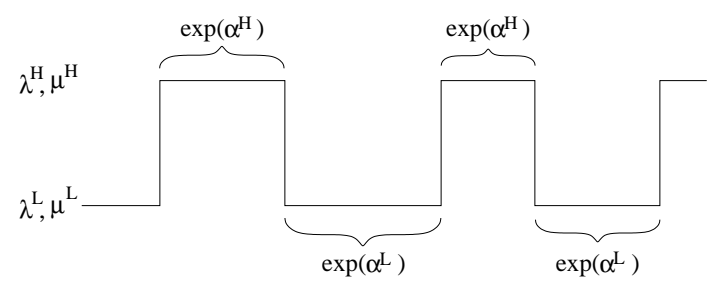

Figure 1: Alternating Load Model

\section{Prior Work}

Time-varying models, have been very widely studied since the earliest work in the 50's, continuing unabated to the present. (In the interest of brevity, we focus on models having non-deterministic switching behavior.) The earliest reference of this type is Clarke [9], who used generating functions to derive expressions for the number in queue. Soon thereafter other researchers applied transforms and generating functions to related models: Neuts [20], Çinlar [7, 8], Arjas [3]. Yechiali and Naor [34] used generating functions to reduce the solution of our model to that of obtaining the roots of a cubic equation. Using similar techniques, de Smit [10] obtained a Weiner-Hopf factorization for systems with MAP arrivals and general service; Sengupta [29] analyzed a system with Markovian arrival and service distributions and service interruptions; Takine and Sengupta [32] generalized [29] to MAP arrivals and general service; Adan and Kulkarni [2] allowed dependencies between successive arrivals and services in a MAP/G/1 framework; and finally Harrison and Zatschler [12] numerically derived the entire sojourn time distribution for very general Markovian systems which they call G-Queues.

A second class of highly effective analytical tools for timevarying models are the Matrix Analytical and related techniques. Neuts [21] used Matrix Analytical techniques to obtain numerical results for our model, observing that its behavior could be qualitatively different from the stationary $M / M / 1$; Ramaswami [25] allowed general service times and Markovian Arrival Processes (MAP); Lucontoni, MeierHellstern and Neuts [15] modeled phase-type service and added server vacations; Sengupta [30] allowed dependencies between semi-Markov interarrival and semi-Markov service times; Takine et al. [31] combined Matrix Analytical techniques with generating functions to allow multiple customer classes and priorities; Lucantoni and Neuts [16] allowed batch MAP arrivals; Mitrani and Chakka numerically compared Matrix Analytic and spectral expansion techniques [18]; and finally Asmussen and Møller [4] solved matrix equations to get the entire waiting time distribution for a queue with MAP arrivals, phase-type service and multiple servers.

It is thus clear that researchers have been highly effective at developing methods to obtain numerical results, but what about basic properties, intuitive insights and simple approximations? Researchers have been at work in these directions as well. One of the classic conjectures in queueing theory was posed by Ross [28], who conjectured that increasing variability in (fluctuation rate) in a Poisson arrival process would (weakly) increase mean customer delay, when the service rate does not vary. Rolski [27] confirmed this conjecture, and more recently Miyoshi and Rolski [19] extended the proof of Ross's conjecture to more general queueing mod- els. Heyman [13] provided a contrasting insight - he gave an example of a deterministically varying arrival function that performs no worse than the stationary version. We continue this tradition in our current work - generalizing [13] by finding simple conditions which guarantee that a stationary system and its time-varying analog perform identically in our Markovian setting.

Another way to garner intuition for time-varying systems is to analyze limiting regimes. Very early on, Newell $[22,23$, 24] used diffusion approximations for time-varying $M / M / 1$ queues. Later, Massey [17] used uniform acceleration to derive the transient behaviors; Abate, Choudhury and Whitt [1] derived tail asymptotics for the waiting time and workload in MAP/GI/1 and MAP/MSP/1 queues; and Rider [26], Gelenbe and Rosenberg [11], Choudhury et al. [6], and Yang and Knessl [33] evaluated the special case when transitions happen much more slowly than arrivals or departures. Finally, Knessl and Yang [14] restricted themselves to a case in which the traffic intensity takes a very specific form, with the aim of generating insights for more general cases.

\section{Our Goals}

As we saw above, the prior work is very effective at producing computational results for our, and even more complex models. However, it is more limited at providing intuition. Part of the problem is that all these methods (generating functions, Matrix Analytical, Spectral Expansion) involve calculating the root of a cubic equation. While in theory a cubic polynomial can be solved analytically, in practice the solution is so cumbersome (dozens of lines in Mathematica) that there is no way to get a sense of the effect of the input parameters on the system performance. For example, the prior work does not provide a sense of the shape of the response time curve, nor how response time relates to the input primitives, such as the $\alpha^{H}$ and $\alpha^{L}$ parameters or the $\lambda^{H}, \lambda^{L}, \mu_{H}, \mu_{L}$ parameters. Our goal in this paper is to get this type of intuition.

One of the simplest/most fundamental questions is what happens when the rate of fluctuation (the $\alpha$ 's) either approach zero or approach infinity. The prior work has not yet provided answers to even the very basic question of whether fast or slow fluctuations lead to higher mean response times. Ross [28] conjectured, and Rolski confirmed [27], that fluctuation leads to higher mean response time for the case where the mean service rate is a constant $\left(\mu^{H}=\mu^{L}=\mu\right)$. In our more general model, however, where the service rate also changes $\left(\mu^{H} \neq \mu^{L}\right)$, we find in Section 2 that higher rate of fluctuation does not always lead to higher mean response time. There are cases where the response time is insensitive to the rate of fluctuation, or can even drop as we increase the rate of fluctuation. We derive a criterion, based on the notion of "slack," $\left(s^{H}\right.$ and $\left.s^{L}\right)$ where $s^{H}=\mu^{H}-\lambda^{H}$ and $s^{L}=\mu^{L}-\lambda^{L}$, which determines whether faster or slower rates of fluctuation result in better system performance.

Another fundamental question in the same vein is whether response time is always bounded by the two asymptotes, the case of high fluctuation rate and low fluctuation rate. Specifically, does a system with a "medium" fluctuation rate always have mean response time in between those two extreme cases? Does mean response time change monotonically between those two extremes? We answer these questions affirmatively in Section 4, where we prove monotonicity results.

While our results thus far have dealt with the overall time- 
average mean response time, it is also of practical importance to understand how this time-average mean compares to the experience of a customer arriving into a "high" $(H)$ period or a customer arriving into a "low" $(L)$ period. Once again computational results can be used to evaluate specific instances, however we seek a qualitative ordering. We answer this question in Section 6, comparing three quantities: the number in system witnessed by an arrival into an $H$ period, the number in system witnessed by an arrival into an $L$ period, and the number in system witnessed by an arrival into a stationary system whose arrival rate is the weighted average of the two arrival rates and whose service rate is the weighted average of the two service rates. We find that, surprisingly, a stochastic dominance relationship exists. However, counter to intuition we find that while the number of jobs seen by an arrival into the 'average' system and the number of jobs seen by an arrival into an $L$ phase are both stochastically dominated by the number of jobs seen by an arrival into an $H$ phase, the number of jobs seen by an arrival into an $L$ phase is not stochastically dominated by the number of jobs in the average system.

Finally our work produces simple and accurate approximations for mean response time and the z-transform of the number of jobs in the system. We provide both a simple closed-form approximation which holds for all fluctuation rates $(\alpha$ 's), as well as even simpler approximations which specialize for the case of only "high" or "low" $\alpha$. While computational methods exist for obtaining the exact mean response time, our simple and accurate approximations have many advantages over the exact results. From a computational perspective, the fact that our approximations are closed-form solutions means that they can easily be computed on any spread-sheet. More importantly, however, our approximations provide the first results about the shape of the mean response time curve as a function of the fluctuation rate, $\alpha$. In particular, they provide a simple and accurate approximation for the curve's functional form. The advantage of the simple functional form is that it shows which primitives are most important in determining mean response time, and allows for further sensitivity analysis.

Throughout the majority of the paper we investigate the characteristics of the mean number in system, $E[N]$, as through application of Little's Law (using the time-average arrival rate) this yields results for mean response time.

\section{ANOMALOUS BEHAVIOR OF FLUCTU- ATING LOAD QUEUE}

We start our work by asking the most basic of questions: How does the mean number of jobs in the system, $E[N]$, compare in the case when the load fluctuates slowly (low $\alpha$ ), as compared with the case where the load fluctuates quickly (high $\alpha$ )? For all the work that has been done on numerically evaluating instances of our model, the question of whether $E[N]$ is higher under low $\alpha$ or high $\alpha$ has not been addressed. Although intuition would tell us that low $\alpha$ should lead to higher $E[N]$ because there is seemingly more variability in the load in this case, this fact has not been proven. In this section we prove that lower $\alpha$ does not always lead to higher $E[N]$, and we derive a criterion that tells us when $E[N]$ increases for low $\alpha$ and when it decreases for low $\alpha$. Before we can state our theorem, we need to define a quantity which we call slack and which we use throughout the paper.
Definition 1. The slack during the low load period is defined as $s^{L} \equiv \mu^{L}-\lambda^{L}$. The slack during the high load period is defined as $s^{H} \equiv \mu^{\dot{H}}-\lambda^{H}$.

In our model we make no assumptions about $\mu^{L}, \mu^{H}, \lambda^{L}$, or $\lambda^{H}$, except to assume that $\rho^{H} \equiv \frac{\lambda^{H}}{\mu^{H}}>\rho^{L} \equiv \frac{\lambda^{L}}{\mu^{L}}$. We allow $\rho^{H}>1$, so long as stability is met. The remainder of the section will be spent proving Theorem 1 below; providing a condition for stability; and discussing the nebulous concept of "load," in a load-fluctuating system.

THEOREM 1. Let $\alpha=\alpha^{L}+\alpha^{H}$. If $s^{L}<s^{H}$, then $E\left[N^{\alpha \rightarrow 0}\right]<E\left[N^{\alpha \rightarrow \infty}\right]$. If $s^{L}>s^{H}$, then $E\left[N^{\alpha \rightarrow 0}\right]>E\left[N^{\alpha \rightarrow \infty}\right]$. If $s^{L}=s^{H}$, then $E\left[N^{\alpha \rightarrow 0}\right]=E\left[N^{\alpha \rightarrow \infty}\right]$.

Corollary 1. If $\mu^{H}=\mu^{L}$, then $E\left[N^{\alpha \rightarrow 0}\right] \geq E\left[N^{\alpha \rightarrow \infty}\right]$ for all settings. This confirms Ross's Conjecture.

We start with a discussion of the two extreme values of $E[N]$ - the case where $\alpha^{L}$ and $\alpha^{H}$ are both very low, and that where $\alpha^{L}$ and $\alpha^{H}$ are both very high. When the $\alpha$ 's are very low, $E[N]$ can be shown to be a weighted mixture of the mean numbers of jobs under two stationary $M / M / 1$ queues: one with load $\rho^{L}$ and the other with load $\rho^{H}$. This may seem obvious, but it will be formally verified via our analysis in Section 3. Specifically, we have:

$$
E\left[N^{\alpha \rightarrow 0}\right]=\frac{\frac{\rho^{L}}{1-\rho^{L}} \cdot \frac{1}{\alpha^{L}}+\frac{\rho^{H}}{1-\rho^{H}} \cdot \frac{1}{\alpha^{H}}}{\frac{1}{\alpha^{L}}+\frac{1}{\alpha^{H}}}
$$

By contrast, when $\alpha^{L}$ and $\alpha^{H}$ are very high, fluctuations are very rapid. In this case, our analysis in Section 3 will show that the system converges to a single $M / M / 1$ queue with load $\rho^{A}$ where,

$$
\rho^{A}=\frac{\lambda^{A}}{\mu^{A}}=\frac{\frac{\lambda^{H}}{\alpha^{H}}+\frac{\lambda^{L}}{\alpha^{L}}}{\frac{\mu^{H}}{\alpha^{H}}+\frac{\mu^{L}}{\alpha^{L}}}
$$

and

$$
E\left[N^{\alpha \rightarrow \infty}\right]=\frac{\rho^{A}}{1-\rho^{A}}
$$

Observation 1. We observe that $\rho^{A}$ as defined above serves as a stability criterion for the system under all $\alpha^{H}$ and $\alpha^{L}$ values, since $\rho^{A}<1$ is equivalent to saying that the timeaverage arrival rate is less than the time-average service rate. However, $\rho^{A}$ does not represent the true load. Specifically

$$
\rho^{A} \neq 1-\pi_{0}
$$

where $\pi_{0}$ represents the fraction of time that the system is idle. In fact, we conjecture that determining $\pi_{0}$ is as hard a problem as determining $E[N]$.

We now prove Theorem 1.

Proof. The necessary and sufficient condition for $E\left[N^{\alpha \rightarrow 0}\right]<E\left[N^{\alpha \rightarrow \infty}\right]$ is:

$$
\frac{\frac{\rho^{L}}{\left(1-\rho^{L}\right) \alpha_{L}}+\frac{\rho^{H}}{\left(1-\rho^{H}\right) \alpha_{H}}}{\frac{1}{\alpha_{L}}+\frac{1}{\alpha_{H}}}<\frac{\rho^{A}}{1-\rho^{A}}
$$

which reduces to

$$
\lambda_{H}(1-c)+\lambda_{L}\left(1-\frac{1}{c}\right)>0
$$




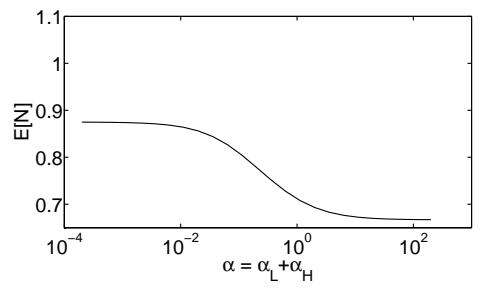

(a) $s^{L}>s^{H}$

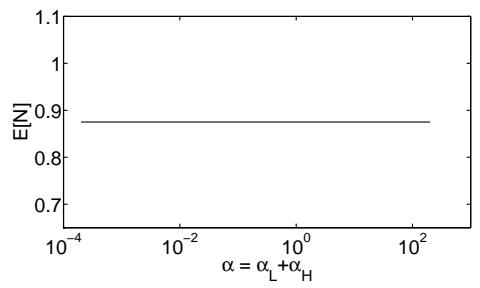

(b) $s^{L}=s^{H}$

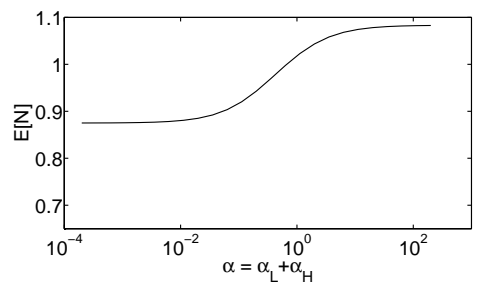

(c) $s^{L}<s^{H}$

Figure 2: Illustration of the anomalous behavior of $\mathrm{E}[\mathrm{N}]$ as a function of $\alpha$, described in Theorem 1. For all three figures, we fix $\rho^{L}=0.2$ and $\rho^{H}=0.6$, and $\alpha^{L}=\alpha^{H}$. The rest of the parameters are as follows: (a) $\mu^{L}=1$, $\lambda^{L}=0.2, \mu^{H}=1, \lambda^{H}=0.6$; (b) $\mu^{L}=1, \lambda^{L}=0.2, \mu^{H}=2, \lambda^{H}=1.2$; (c) $\mu^{L}=1, \lambda^{L}=0.2, \mu^{H}=4, \lambda^{H}=2.4$.

where $c=\frac{\mu_{L}-\lambda_{L}}{\mu_{H}-\lambda_{H}}$. Or,

$$
\lambda_{H} c^{2}-c\left(\lambda_{H}+\lambda_{L}\right)+\lambda_{L}<0
$$

The solution to above inequality is $c \in\left(\frac{\lambda_{L}}{\lambda_{H}}, 1\right)$. Note that $c>\frac{\lambda_{L}}{\lambda_{H}}$ is equivalent to $\rho^{H}>\rho^{L}$, which is trivially true. Therefore the only other condition is $c<1$, or $\left(\mu_{L}-\lambda_{L}\right)<$ $\left(\mu_{H}-\lambda_{H}\right)$. Note, we are assuming $\rho^{H}<1$, otherwise this behavior is not possible. The remaining cases in the theorem are proven analogously.

The anomalous behavior of the fluctuating-load queue is illustrated in Figure 2.

\section{ANALYSIS}

Our approach is based on calculating the $z$-transform for the distribution of number of jobs at instants when the system switches from a Low $(L)$ phase to a High $(H)$ phase and when the system switches from an $H$ phase to an $L$ phase. In Section 3.1 we prove that knowledge of the distributions at switching points suffices to determine the distribution of the number of jobs in the system at a randomly sampled point in time. The remainder of the section will derive the transform of the number of jobs at the end of the $L$ phase, $\widehat{\Pi}^{L}(z)$, and that at the end of the $H$ phase, $\widehat{\Pi^{H}}(z)$. To derive these, we will first obtain a transition function which maps the distribution of number of jobs at a switching point to the distribution at the next switching point (Section 3.2, equation (3)). This transition function will then allow us to express $\widehat{\Pi^{L}}(z)$ in terms of $\widehat{\Pi^{H}}(z)$, and vice-versa (see Section 3.3, Equations (4-5)). Finally we solve these to get expressions for $\widehat{\Pi}^{L}(z)$ and $\widehat{\Pi^{H}}(z)$ in terms of $\pi_{0}$ only (see Section 3.3, Equation (9) ). All the transform derivations described above will assume a more general model than we have considered so far, where we allow for a burst of arrivals at each arrival instant, where the burst size can be arbitrary.

\subsection{Conditional PASTA}

Let $N^{L}$ and $N^{H}$ be the random variables for the number of jobs at the end of $L$ and $H$ phases respectively 3 . If $N$ denotes the random variable for the number of jobs in the system at a randomly sampled point, the following theorem relates the distribution of $N$ with those of $N^{L}$ and $N^{H}$.

TheOREM 2. $N$ has the same distribution as $\mathscr{N}$, where

$$
\mathscr{N}= \begin{cases}N^{L} & \text { w.p. } \frac{\alpha^{H}}{\alpha^{L}+\alpha^{H}} \\ N^{H} & \text { w.p. } \frac{\alpha^{L}}{\alpha^{L}+\alpha^{H}}\end{cases}
$$

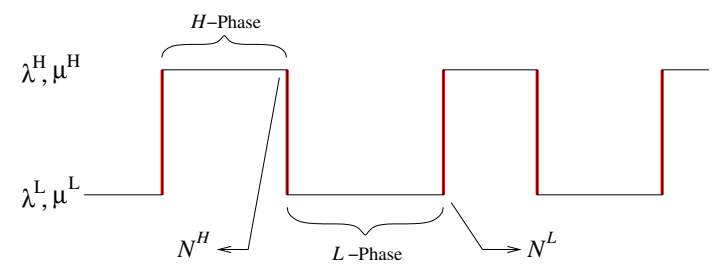

Figure 3: Switching Points used in Analysis

ProOF. Let $\widehat{\Pi^{L}}(z), \widehat{\Pi^{H}}(z)$ and $\widehat{\Pi}(z)$ be the $z$-transforms of $N^{L}, N^{H}$ and $N$ respectively. Proving the above theorem is equivalent to proving

$$
\widehat{\Pi}(z)=\frac{\frac{\widehat{\Pi^{L}}(z)}{\alpha^{L}}+\frac{\widehat{\Pi^{H}}(z)}{\alpha^{H}}}{\frac{1}{\alpha^{L}}+\frac{1}{\alpha^{H}}}
$$

Let $\widehat{\Pi^{L}}(z, t)$ be the $z$-transform for the number of jobs in the system $t$ units of time after the start of the $L$ phase, conditioned on the phase being longer than $t$, and let $\widehat{\Pi^{H}}(z, t)$ be the corresponding quantity for $H$ phase (Note that $\widehat{\Pi^{L}}(z, 0)=$ $\left.\widehat{\Pi^{H}}(z)\right)$. We will use renewal-reward theory to prove the above equality where a cycle consists of a single $L$ phase followed by a single $H$ phase, and where $r(T)=z^{n(T)}$ is the instantaneous reward earned at time $T, n(T)$ being the number of jobs at time $T$. Clearly, $\widehat{\Pi}(z)$ is the long run average rate at which reward is earned. Therefore,

$$
\begin{aligned}
\widehat{\Pi}(z)= & \frac{E[\text { reward in } L \text { phase }]+E[\text { reward in } H \text { phase }]}{E[\text { length of } L \text { phase }]+E[\text { length of } H \text { phase }]} \\
= & \left(\frac{1}{\alpha^{L}}+\frac{1}{\alpha^{H}}\right)^{-1}\left[\int_{t=0}^{\infty} \int_{u=0}^{t} \widehat{\Pi^{L}}(z, u) d u \alpha_{L} e^{-\alpha^{L} t} d t\right. \\
& \left.+\int_{t=0}^{\infty} \int_{u=0}^{t} \widehat{\Pi^{H}}(z, u) d u \alpha^{H} e^{-\alpha^{H} t} d t\right] \\
= & \left(\frac{1}{\alpha^{L}}+\frac{1}{\alpha^{H}}\right)^{-1}\left[\int_{u=0}^{\infty} \int_{t=u}^{\infty} \widehat{\Pi^{L}}(z, u) d u \alpha^{L} e^{-\alpha^{L} t} d t\right. \\
& \left.+\int_{u=0}^{\infty} \int_{t=u}^{\infty} \widehat{\Pi^{H}}(z, u) d u \alpha^{H} e^{-\alpha^{H} t} d t\right] \\
= & \frac{\frac{\widehat{\Pi}^{L}(z)}{\alpha^{L}}+\frac{\widehat{\Pi^{H}}(z)}{\alpha^{H}}}{\alpha^{L}}+\frac{1}{\alpha^{H}}
\end{aligned}
$$

The intuition behind Theorem 2 is the PASTA (Poisson Arrivals See Time Averages) property exhibited by the 
Markovian switching process, as we prove next.

THEOREM 3. The time average distribution of number of jobs in the system during $L$ phases (respectively $H$ phases) is the same as the distribution of $N^{L}$ (respectively $N^{H}$ ).

Proof. Consider any general queueing system where we set off timers after $\exp \left(\alpha^{L}\right)$ units of time. Whenever a timer expires, we restart the system with some number of jobs sampled from the distribution of $N^{H}$. This can be visualized as seeing only the $L$ phases of the original queueing system stitched together. Since the timer events are a Poisson process, by PASTA, the distribution of number of jobs at these event instants is the same as the time average distribution which is the time average distribution of number of jobs during the $L$ phase in the original system.

Now, since the long term fraction of time spent in $L$ phases is $\frac{\alpha^{H}}{\alpha^{L}+\alpha^{H}}$ and in $H$ is $\frac{\alpha^{L}}{\alpha^{L}+\alpha^{H}}$, the linear combination of Theorem 2 follows. Although we have proved the above result for only one observable quantity, the number of jobs in the system, the result holds for any observable quantity e.g. square of number of jobs in system, age of the job in service, $z$-transform of the number of jobs in service.

To summarize, although we defined $N^{L}$ and $N^{H}$ to be the distributions of number of jobs at switching points, they are the same as the distributions for number of jobs seen by an arbitrary arrival during the $L$ or $H$ phase, respectively.

\subsection{Derivation of Transition functions}

Our goal in this section is to derive a transition function which maps the distribution of the number of jobs at a switching point to the distribution at the next switching point. To do this, we first need to return to a simple $M / M / 1$ queue (without fluctuating load), and consider its transient behavior with respect to the number of jobs at time $T \sim \exp (\alpha)$, given a distribution on the number of jobs at time 0 .

The analysis that follows is motivated by Bailey's [5] work on transient analysis of $M / M / 1$ queues. We will consider an $M / M / 1$ queue with service rate $\mu$ where with rate $\lambda$ arrivals occur with burst size distribution having (known) $z$-transform $\widehat{A}(z)$. Let $a_{i}$ be the probability that the burst size is $i$ (wlog, $\left.a_{0}=0\right)$. Also, let $p_{i}(t)$ be the probability that there are $i$ jobs in the system at time $t$. Define

$$
\widehat{\Pi}(z, t)=\sum_{i=0}^{\infty} p_{i}(t) z^{i}
$$

We can now write the differential equations for this system:

$$
\begin{aligned}
& \frac{d p_{i}(t)}{d t}=\lambda \sum_{j=1}^{i} a_{j} p_{i-j}(t)-(\lambda+\mu) p_{i}(t)+\mu p_{i+1}(t) \\
& \frac{d p_{0}(t)}{d t}=-\lambda p_{0}(t)+\mu p_{1}(t)
\end{aligned}
$$

which gives,

$$
\begin{aligned}
\frac{\partial \widehat{\Pi}(z, t)}{\partial t} & =\widehat{\Pi}(z, t)\{\mu(1-z)-\lambda(1-\widehat{A}(z))\} \\
& -\mu(1-z) p_{0}(t)
\end{aligned}
$$

Let $T$ denote an exponentially distributed random variable with mean $\frac{1}{\alpha}$, and let $\widehat{\Pi}_{\alpha}(z)=\widehat{\Pi}(z, T)$. Then,

$$
\begin{aligned}
\widehat{\Pi}_{\alpha}(z) & =\int_{0}^{\infty} \widehat{\Pi}(z, t) \alpha e^{-\alpha t} d t \\
& =\frac{\alpha z \widehat{\Pi}(z, 0)-\mu(1-z) p_{0}(T)}{\alpha z-\mu(1-z)+\lambda z(1-\widehat{A}(z))}
\end{aligned}
$$

To complete the solution we need to find $p_{0}(T)$. Note that the denominator of $(3)$ has a root $\xi \in(0,1)$. For $\widehat{\Pi}_{\alpha}(z)$ to converge inside the unit disk $|z|<1, \xi$ must also be a root of the numerator. Hence,

$$
p_{0}(T)=\frac{\alpha \xi \widehat{\Pi}(\xi, 0)}{\mu(1-\xi)}
$$

The transition functions mapping the distribution at the start of an $L$ or an $H$ phase to the end of the phase is obtained by setting $\mu, \lambda, \alpha$ and $\widehat{A}(z)$ appropriately in (3).

\subsection{Distributions at Switching Points}

Let $\widehat{A^{L}}(z)$ (respectively $\left.\widehat{A^{H}}(z)\right)$ be the $z$-transform for the distribution of the burst sizes for arrivals during $L$ (respectively $H$ ). Let $\overline{A^{L}}$ and $\overline{A^{H}}$ be the mean burst sizes during $L$ and $H$ phases. For convenience, we also define the following

$$
\begin{aligned}
\widehat{B^{L}}(z) & =\frac{1-\widehat{A^{L}}(z)}{1-z} \\
\widehat{B^{H}}(z) & =\frac{1-\widehat{A^{H}}(z)}{1-z} \\
\sigma_{z}^{L} & =\mu^{L}-\lambda^{L} z \widehat{B^{L}}(z) \\
\sigma_{z}^{H} & =\mu^{H}-\lambda^{H} z \widehat{B^{H}}(z) \\
\sigma^{L} & =\left.\sigma_{z}^{L}\right|_{z=1}=\mu^{L}-\lambda^{L} \overline{A^{L}} \\
\sigma^{H} & =\left.\sigma_{z}^{H}\right|_{z=1}=\mu^{H}-\lambda^{H} \overline{A^{H}}
\end{aligned}
$$

From (3), we can write the following relations

$$
\begin{aligned}
& \widehat{\Pi^{L}}(z)=\frac{\alpha^{L} z \widehat{\Pi^{H}}(z)-\mu^{L}(1-z) \pi_{0}^{L}}{\alpha^{L} z-\mu^{L}(1-z)+\lambda^{L} z\left(1-\widehat{A^{L}}(z)\right)} \\
& \widehat{\Pi^{H}}(z)=\frac{\alpha^{H} z \widehat{\Pi^{L}}(z)-\mu^{H}(1-z) \pi_{0}^{H}}{\alpha^{H} z-\mu^{H}(1-z)+\lambda^{H} z\left(1-\widehat{A^{H}}(z)\right)}
\end{aligned}
$$

where $\pi_{0}^{L}=\operatorname{Pr}\left\{N^{L}=0\right\}$ and $\pi_{0}^{H}=\operatorname{Pr}\left\{N^{H}=0\right\}$ are unknowns. We can solve (4)-(5) for $\widehat{\Pi}^{L}(z)$ to get

$$
\widehat{\Pi^{L}}(z)=\frac{z \alpha^{L} \mu^{H} \pi_{0}^{H}+z \alpha^{H} \mu^{L} \pi_{0}^{L}-(1-z) \sigma_{z}^{H} \mu^{L} \pi_{0}^{L}}{z \alpha^{H} \sigma_{z}^{L}+z \alpha^{L} \sigma_{z}^{H}-(1-z) \sigma_{z}^{H} \sigma_{z}^{L}}
$$

By substituting $z=1$ in the above equation we get one equation relating $\pi_{0}^{L}$ and $\pi_{0}^{H}$ :

$$
\begin{aligned}
\frac{\frac{\mu^{L}}{\alpha^{L}} \pi_{0}^{L}+\frac{\mu^{H}}{\alpha^{H}} \pi_{0}^{H}}{\frac{\mu^{L}}{\alpha^{L}}+\frac{\mu^{H}}{\alpha^{H}}} & =1-\frac{\frac{\lambda^{L} \overline{A^{L}}}{\alpha^{L}}+\frac{\lambda^{H} \overline{A^{H}}}{\alpha^{H}}}{\frac{\mu^{L}}{\alpha^{L}}+\frac{\mu^{H}}{\alpha^{H}}} \\
& =1-\rho^{A}
\end{aligned}
$$

It turns out that there is a very simple explanation for (7) based on Little's Law. Imagine stretching the $L$ periods by a factor of $\mu^{L}$ and $H$ periods by $\mu^{H}$. Now in this transformed time, the service rate is a constant, 1 . The switching times are distributed as $\exp \left(\alpha^{L} / \mu^{L}\right)$ and $\exp \left(\alpha^{H} / \mu^{H}\right)$. The time average arrival rate scales by a factor of $\left(\frac{1}{\alpha^{L}}+\frac{1}{\alpha^{H}}\right)$ divided 
by $\left(\frac{\mu^{L}}{\alpha^{L}}+\frac{\mu^{H}}{\alpha^{H}}\right)$, because the arrivals that were earlier occurring in $\left(\frac{1}{\alpha^{L}}+\frac{1}{\alpha^{H}}\right)$ now occur in $\left(\frac{\mu^{L}}{\alpha^{L}}+\frac{\mu^{H}}{\alpha^{H}}\right)$ amount of time. Applying Little's Law at the server gives (7).

To complete the solution, we need one more equation relating $\pi_{0}^{L}$ and $\pi_{0}^{H}$. Using PASTA we know that

$$
\frac{\frac{\pi_{0}^{L}}{\alpha^{L}}+\frac{\pi_{0}^{H}}{\alpha^{H}}}{\frac{1}{\alpha^{L}}+\frac{1}{\alpha^{H}}}=\pi_{0}
$$

where $\pi_{0}$ is the long term fraction of the time when system is idle. However, $\pi_{0}$ is also unknown and is not equal to $1-\rho^{A}$. But, by using (7), (6) simplifies to

$$
\widehat{\Pi^{L}}(z)=\frac{z \alpha^{H} \sigma^{L}+z \alpha^{L} \sigma^{H}-(1-z) \sigma_{z}^{H}\left(\mu^{L} \pi_{0}^{L}\right)}{z \alpha^{H} \sigma_{z}^{L}+z \alpha^{L} \sigma_{z}^{H}-(1-z) \sigma_{z}^{H} \sigma_{z}^{L}}
$$

The expression for $\widehat{\Pi^{H}}(z)$ is completely symmetric to (9). In the rest of the paper we will analyze a special case of the above queueing system where $\widehat{A^{L}}(z)=\widehat{A^{H}}(z)=z$ (burst size $\equiv 1)$. For this case the $z$-transforms become

$$
\begin{aligned}
\widehat{\Pi^{H}}(z) & =\frac{z \alpha^{H} s^{L}+z \alpha^{L} s^{H}-(1-z) s_{z}^{L}\left(\mu^{H} \pi_{0}^{H}\right)}{z \alpha^{H} s_{z}^{L}+z \alpha^{L} s_{z}^{H}-(1-z) s_{z}^{H} a_{z}^{L}} \\
\widehat{\Pi^{L}}(z) & =\frac{z \alpha^{H} s^{L}+z \alpha^{L} s^{H}-(1-z) s_{z}^{H}\left(\mu^{L} \pi_{0}^{L}\right)}{z \alpha^{H} s_{z}^{L}+z \alpha^{L} s_{z}^{H}-(1-z) s_{z}^{H} s_{z}^{L}}
\end{aligned}
$$

where,

$$
\begin{array}{ll}
s_{z}^{L}=\mu^{L}-\lambda^{L} z, & s_{z}^{H}=\mu^{H}-\lambda^{H} z \\
s^{L}=\mu^{L}-\lambda^{L}, & s^{H}=\mu^{H}-\lambda^{H}
\end{array}
$$

These expressions agree with those derived by Yechiali and Naor [34]. Again, we can solve for $\pi_{0}^{L}$ and $\pi_{0}^{H}$ by noticing that the cubic polynomial in the denominators of these expressions has a root in $(0,1)$ where the both the numerators must also be 0 . Solving for this root, however, does not achieve our goals of getting simple and intuitive expressions.

\section{RESULTS - MONOTONICITY OF $E[N]$}

In Section 2, we analyzed how asymptotic behavior of our 2-phase fluctuating load queue compares for the cases $\alpha \rightarrow 0$ and $\alpha \rightarrow \infty$. We are now interested in seeing the behavior in the mid- $\alpha$ range. To be precise, we want to answer questions like: If we fix the values of $\mu^{L}, \mu^{H}, \lambda^{L}, \lambda^{H}$ and the ratio $\frac{\alpha^{L}}{\alpha^{H}}$, but start increasing $\alpha\left(\alpha=\alpha^{H}+\alpha^{L}\right)$ from 0 to $\infty$, how do $E[N], E\left[N^{L}\right]$ and $E\left[N^{H}\right]$ behave? Are they always between the asymptotes of Section 2? Do they increase or decrease monotonically with $\alpha$ ? What is the asymptotic behavior when $\rho^{H}>1$ ?

We will first derive expressions for $\pi_{0}^{L}$ and $\pi_{0}^{H}$ as linear combinations of the asymptotes, and show that these are monotonic, in Section 4.1. Then, in Section 4.2, we will introduce $r$, the parameterization we will use for our ultimate expressions for $E[N]$, recasting the expressions in 4.1 in terms of $r$. We will then use these expressions to prove the desired monotonicity results for $E[N]$, first for $\rho^{H}<1$, in Section 4.3, and then in Section 4.4 for $\rho^{H}>1$. These latter results will be attained by expressing the mean number in system for a given instance in terms of the parameter $r$, which can be thought of as denoting the ratio, or the mixture, of the two asymptotes at the given parameter setting.

Throughout, we disregard the singular case $\rho^{H}=1$.

\subsection{Monotonicity of the $\pi_{0}$ 's}

Our first step is to derive expressions for $\pi_{0}^{L}$ and $\pi_{0}^{H}$. To ease our derivation, we define $\Delta=\frac{\left(\alpha^{L}+\alpha^{H}\right) \mu^{A}}{\mu^{L} \mu^{H}}$ (we will assume $\left.\mu^{L}, \mu^{H} \neq 0\right)$. The intuition behind $\Delta$ instead of $\alpha$ is that $\Delta$ can be viewed as normalizing the scale of the problem so that further analysis only depends on $\rho^{A}, \rho^{L}$ and $\rho^{H}$.

Using $\Delta$, Equations (10)-(11), can be rewritten as follows:

$$
\begin{aligned}
\widehat{\Pi^{H}}(z) & =\frac{z \Delta\left(1-\rho^{A}\right)-(1-z)\left(1-\rho^{L} z\right) \pi_{0}^{H}}{z \Delta\left(1-\rho^{A} z\right)-(1-z)\left(1-\rho^{L} z\right)\left(1-\rho^{H} z\right)} \\
\widehat{\Pi^{L}}(z) & =\frac{z \Delta\left(1-\rho^{A}\right)-(1-z)\left(1-\rho^{H} z\right) \pi_{0}^{L}}{z \Delta\left(1-\rho^{A} z\right)-(1-z)\left(1-\rho^{L} z\right)\left(1-\rho^{H} z\right)}
\end{aligned}
$$

To get expressions for $\pi_{0}^{L}$ and $\pi_{0}^{H}$, we examine the roots of the denominator of (12) and (13), $f(z)$ :

$$
f(z)=z \Delta\left(1-\rho^{A} z\right)-(1-z)\left(1-\rho^{L} z\right)\left(1-\rho^{H} z\right) .
$$

As mentioned previously, since $f(0)=-1<0$ and $f(1)=$ $\Delta\left(1-\rho^{A}\right)>0, f(z)$ has a root $\chi \in[0,1)$ at which the numerators of $\widehat{\Pi^{H}}(z)$ and $\widehat{\Pi}^{L}(z)$ must be zero for the $z$-transforms to converge inside the unit disk $|z|<1$. Therefore,

$$
\begin{aligned}
\pi_{0}^{H} & =\frac{\chi}{(1-\chi)\left(1-\rho^{L} \chi\right)} \Delta\left(1-\rho^{A}\right) \\
\pi_{0}^{L} & =\frac{\chi}{(1-\chi)\left(1-\rho^{H} \chi\right)} \Delta\left(1-\rho^{A}\right)
\end{aligned}
$$

yielding

$$
\pi_{0}^{H}\left(1-\rho^{L} \chi\right)=\pi_{0}^{L}\left(1-\rho^{H} \chi\right)
$$

Combining this with (7) and introducing $\theta=\left(\frac{1-\rho^{A}}{1-\rho^{A} \chi}\right)$ yields simpler expressions for $\pi_{0}^{H}$ and $\pi_{0}^{L}$ :

$$
\begin{aligned}
\pi_{0}^{H} & =\frac{1}{\rho^{A}}\left[\left(1-\rho^{A}\right) \rho^{H}-\theta\left(\rho^{H}-\rho^{A}\right)\right] \\
\pi_{0}^{L} & =\frac{1}{\rho^{A}}\left[\left(1-\rho^{A}\right) \rho^{L}+\theta\left(\rho^{A}-\rho^{L}\right)\right]
\end{aligned}
$$

We can write the above as follows:

$$
\begin{aligned}
& \pi_{0}^{H}=\left(1-\rho^{A}\right)\left[\frac{1-\theta}{1-\left(1-\rho^{A}\right)}\right]+\left(1-\rho^{H}\right)\left[\frac{\theta-\left(1-\rho^{A}\right)}{1-\left(1-\rho^{A}\right)}\right] \\
& \pi_{0}^{L}=\left(1-\rho^{A}\right)\left[\frac{1-\theta}{1-\left(1-\rho^{A}\right)}\right]+\left(1-\rho^{L}\right)\left[\frac{\theta-\left(1-\rho^{A}\right)}{1-\left(1-\rho^{A}\right)}\right]
\end{aligned}
$$

or equivalently as

$$
\begin{aligned}
\pi_{0}^{H} & =\left(1-\rho^{A}\right)\left[\frac{\omega-\theta}{\omega-\left(1-\rho^{A}\right)}\right]+0\left[\frac{\theta-\left(1-\rho^{A}\right)}{\omega-\left(1-\rho^{A}\right)}\right] \\
\pi_{0}^{L} & =\left(1-\rho^{A}\right)\left[\frac{\omega-\theta}{\omega-\left(1-\rho^{A}\right)}\right] \\
& +\left(\frac{\left(\rho^{H}-\rho^{L}\right)\left(1-\rho^{A}\right)}{\rho^{H}-\rho^{A}}\right)\left[\frac{\theta-\left(1-\rho^{A}\right)}{\omega-\left(1-\rho^{A}\right)}\right]
\end{aligned}
$$

where $\omega=\frac{\rho^{H}\left(1-\rho^{A}\right)}{\left(\rho^{H}-\rho^{A}\right)}$. By observing that $\lim _{\Delta \rightarrow \infty} \theta=(1-$ $\left.\rho^{A}\right)$ and $\lim _{\Delta \rightarrow 0} \theta=\min (1, \omega)$, equations (15)-(16) can be seen as expressing $\pi_{0}^{H}$ and $\pi_{0}^{L}$ as a convex combination of the limiting cases when $\rho^{H}<1$. Similarly, equations (17)-(18) express $\pi_{0}^{H}$ and $\pi_{0}^{L}$ as a convex combination of the limiting cases when $\rho^{H}>1$. 
Since $\chi$ and hence $\theta$ are monotonic in $\Delta^{1}$, this proves the monotonicity in $\pi_{0}^{H}$ and $\pi_{0}^{L}$.

\subsection{Parameterization in Terms of $r$}

While parameterization in terms of $\theta$ is sufficient to show monotonicity of $\pi_{0}^{L}$ and $\pi_{0}^{H}$, parameterization by $\theta$ does not yield the simplest expressions for $E[N]$, which is our ultimate aim. We seek a new parameter $r$ which will allow us to express $E[N]$ as a convex combination of two limits, similar to what we did above for $\pi_{0}^{H}$ and $\pi_{0}^{L}$.

To obtain this $r$ parameter, we first observe that $\theta$ can be written as the root of the following cubic obtained by substituting $\chi=\left(\frac{\theta-\left(1-\rho^{A}\right)}{\rho^{A} \theta}\right)$ in $(14)$ :

$$
\begin{aligned}
g(\theta) & =\left(\rho^{A}\right)^{2} \Delta\left[\theta-\left(1-\rho^{A}\right)\right] \theta \\
& -[\theta-1]\left[\theta\left(\rho^{A}-\rho^{L}\right)+\rho^{L}\left(1-\rho^{A}\right)\right]\left[\theta\left(\rho^{H}-\rho^{A}\right)-\rho^{H}\left(1-\rho^{A}\right)\right]
\end{aligned}
$$

From (19), we can now deduce the following limiting behaviors:

For $\rho^{H}<1$ :

$$
\begin{aligned}
\lim _{\Delta \rightarrow 0} \theta & \equiv \ell_{\left(\rho^{H}<1\right)}=1 \\
\lim _{\Delta \rightarrow 0} \frac{\ell_{\left(\rho^{H}<1\right)}-\theta}{\Delta} & \equiv \ell_{\left(\rho^{H}<1\right)}^{\prime}=\frac{\rho^{A}}{\left(1-\rho^{L}\right)\left(1-\rho^{H}\right)}
\end{aligned}
$$

For $\rho^{H}>1$ :

$$
\begin{aligned}
\lim _{\Delta \rightarrow 0} \theta & \equiv \ell_{\left(\rho^{H}>1\right)}=\frac{\rho^{H}\left(1-\rho^{A}\right)}{\rho^{H}-\rho^{A}} \\
\lim _{\Delta \rightarrow 0} \frac{\ell_{\left(\rho^{H}>1\right)}-\theta}{\Delta} & \equiv \ell_{\left(\rho^{H}>1\right)}^{\prime}=\frac{\rho^{A} \rho^{H}\left(1-\rho^{A}\right)}{\left(\rho^{H}-\rho^{L}\right)\left(\rho^{H}-1\right)\left(\rho^{H}-\rho^{A}\right)}
\end{aligned}
$$

For the case $\rho^{H}<1$, we will now define the desired $r$ parameter as

$$
r=r_{\left(\rho^{H}<1\right)} \equiv \frac{\ell_{\left(\rho^{H}<1\right)}-\theta}{\Delta \ell_{\left(\rho^{H}<1\right)}^{\prime}}
$$

and for the case $\rho^{H}>1$ as

$$
r=r_{\left(\rho^{H}>1\right)} \equiv \frac{\ell_{\left(\rho^{H}>1\right)}-\theta}{\Delta \ell_{\left(\rho^{H}>1\right)}^{\prime}}
$$

Whenever unambiguous, we will suppress the subscripts on $r$.

To express $E\left[N^{H}\right]$ and $E\left[N^{L}\right]$ as convex combinations in terms of $r$, we start by differentiating our transforms $\widehat{\Pi^{H}}(z)$ and $\widehat{\Pi^{L}(z)}$ and set $z=1$, which results in:

$$
\begin{aligned}
& E\left[N^{H}\right]=\frac{\rho^{A}}{1-\rho^{A}}-\frac{\left(1-\rho^{L}\right)\left(1-\rho^{H}-\pi_{0}^{H}\right)}{\Delta\left(1-\rho^{A}\right)} \\
& E\left[N^{L}\right]=\frac{\rho^{A}}{1-\rho^{A}}-\frac{\left(1-\rho^{H}\right)\left(1-\rho^{L}-\pi_{0}^{L}\right)}{\Delta\left(1-\rho^{A}\right)}
\end{aligned}
$$

\footnotetext{
${ }^{1} \chi$ is the root of a polynomial that is the sum of a cubic with roots $1, \frac{1}{\rho^{L}}, \frac{1}{\rho^{H}}$ and a quadratic that is positive in $\left[0, \frac{1}{\rho^{A}}\right]$ and increases uniformly with $\alpha$. Therefore, $f(z)$ increases uniformly in $\left[0, \frac{1}{\rho^{A}}\right]$ as $\alpha$ increases, hence proving monotonicity in $\chi$.
}

By substituting $\pi_{0}^{H}$ and $\pi_{0}^{L}$ in terms of $\theta$ from (15)-(16) into (26)-(27) and collecting the terms dependent on $\Delta$, we can write $E\left[N^{H}\right]$ and $E\left[N^{L}\right]$ for the case $\rho^{H}<1$ as

$$
\begin{aligned}
& E\left[N^{H}\right]=\left[\frac{\rho^{A}}{1-\rho^{A}}\right]+\left[\frac{\rho^{H}}{1-\rho^{H}}-\frac{\rho^{A}}{1-\rho^{A}}\right] r_{\left(\rho^{H}<1\right)} \\
& E\left[N^{L}\right]=\left[\frac{\rho^{A}}{1-\rho^{A}}\right]+\left[\frac{\rho^{L}}{1-\rho^{L}}-\frac{\rho^{A}}{1-\rho^{A}}\right] r_{\left(\rho^{H}<1\right)}
\end{aligned}
$$

Similarly, for the case $\rho^{H}>1$, the expressions for $E\left[N^{H}\right]$ and $E\left[N^{L}\right]$ are

$$
\begin{aligned}
& E\left[N^{H}\right]= {\left[\frac{\rho^{A}}{1-\rho^{A}}\right]+\left[\frac{\rho^{A}}{1-\rho^{A}}+\frac{\rho^{H}}{\rho^{H}-1}\right]\left[\frac{1-\ell_{\left(\rho^{H}>1\right)}}{-\ell_{\left(\rho^{H}<1\right)}^{\prime} \Delta}\right.} \\
&\left.+\left(\frac{\ell_{\left(\rho^{H}>1\right)}^{\prime}}{-\ell_{\left(\rho^{H}<1\right)}^{\prime}}\right) r_{\left(\rho^{H}>1\right)}\right] \\
& E\left[N^{L}\right]=\left[\frac{\rho^{A}}{1-\rho^{A}}\right]+\left[\frac{\rho^{A}}{1-\rho^{A}}-\frac{\rho^{L}}{1-\rho^{L}}\right]\left[\frac{1-\ell_{\left(\rho^{H}>1\right)}}{-\ell_{\left(\rho^{H}<1\right)}^{\prime}}\right. \\
&\left.+\left(\frac{\ell_{\left(\rho^{H}>1\right)}^{\prime}}{-\ell_{\left(\rho^{H}<1\right)}^{\prime}}\right) r_{\left(\rho^{H}>1\right)}\right]
\end{aligned}
$$

Note that for the case $\rho^{H}<1$, we were able to express $E\left[N^{H}\right]$ and $E\left[N^{L}\right]$ in (28)-(29) as the convex combination of two constants. This is not possible when $\rho^{H}>1$ because when $\Delta \rightarrow 0$, the mean number of jobs becomes unbounded. However for this case, we can write $E\left[N^{H}\right]$ (and respectively $\left.E\left[N^{L}\right]\right)$ as a convex combination of two asymptotic functions of $\Delta$ which are only separated by a constant.

From the analysis carried out earlier in Section 3.1, we can get the expression for $E[N]$ as

$$
E[N]=\frac{\frac{E\left[N^{L}\right]}{\alpha^{L}}+\frac{E\left[N^{H}\right]}{\alpha^{H}}}{\frac{1}{\alpha^{L}}+\frac{1}{\alpha^{H}}}
$$

\subsection{Monotonicity of Number in System, $\rho^{H}<1$}

The following Theorem proves monotonicity of $E[N]$ for $\rho^{H}<1$ :

TheOREM 4. For the case $\rho^{H}<1$

(i) $E\left[N^{L}\right]$ decreases and $E\left[N^{H}\right]$ increases monotonically as the switching rates decrease.

(ii) If $s^{L}<s^{H}$ then $E[N]$ decreases monotonically as switching rates decrease, otherwise it increases monotonically.

Proof. For succinctness, we will define the following quantities

$$
\begin{aligned}
N_{\infty} & \equiv \frac{\rho^{A}}{1-\rho^{A}} \\
N_{0} & \equiv \frac{\frac{1}{\alpha^{H}} \frac{\rho^{H}}{1-\rho^{H}}+\frac{1}{\alpha^{L}} \frac{\rho^{L}}{1-\rho^{L}}}{\frac{1}{\alpha^{H}}+\frac{1}{\alpha_{L}}}
\end{aligned}
$$

From (28)-(29), we can write the expectations of number of jobs in system as

$$
\begin{aligned}
E\left[N^{H}\right] & =\left[\frac{\rho^{A}}{1-\rho^{A}}\right]+\left[\frac{\rho^{H}}{1-\rho^{H}}-\frac{\rho^{A}}{1-\rho^{A}}\right] r \\
E\left[N^{L}\right] & =\left[\frac{\rho^{A}}{1-\rho^{A}}\right]+\left[\frac{\rho^{L}}{1-\rho^{L}}-\frac{\rho^{A}}{1-\rho^{A}}\right] r \\
E[N] & =N_{\infty}+\left[N_{0}-N_{\infty}\right] r
\end{aligned}
$$


From the limits proved in (20)-(21), we know that for the case $\rho^{H}<1, \lim _{\Delta \rightarrow \infty} r=0$ and $\lim _{\Delta \rightarrow 0} r=1$. We will now show that $r$ is monotonic in $\Delta$. This will imply that $E\left[N^{H}\right]$ increases and $E\left[N^{L}\right]$ decreases monotonically as switching rates decrease and $E[N]$ will increase or decrease depending on whether $s^{L}$ is larger or smaller than $s^{H}$, respectively.

By substituting $\theta=1-r \Delta \ell_{\left(\rho^{H}<1\right)}^{\prime}$ in $g(\theta)$ from (19), we get the following polynomial relating $\Delta$ and $r$ :

$$
\begin{aligned}
h(\Delta, r)= & r^{2} \Delta^{2}\left(\ell_{\left(\rho^{H}<1\right)}^{\prime}\right)^{2}\left[\rho^{A}\left(1-\rho^{L}\right)\left(1-\rho^{H}\right)\right. \\
& \left.+r\left(\rho^{A}-\rho^{L}\right)\left(\rho^{H}-\rho^{A}\right)\right] \\
- & r \Delta \ell_{\left(\rho^{H}<1\right)}^{\prime} \rho^{A}\left[\left(1+\rho^{A}\right)\left(1-\rho^{L}\right)\left(1-\rho^{H}\right)\right. \\
& \left.+r\left\{\left(\rho^{A}-\rho^{L}\right)\left(\rho^{H}-1\right)+\left(1-\rho^{L}\right)\left(\rho^{H}-\rho^{A}\right)\right\}\right] \\
& +\left(\rho^{A}\right)^{2}\left(1-\rho^{L}\right)\left(1-\rho^{H}\right)(1-r)
\end{aligned}
$$

The above may be viewed as a cubic for $r$ in terms of $\Delta$, or, alternatively, as a quadratic for $\Delta$ in terms of $r$. Therefore for any $r$ there can be at most two values of $\Delta$. Since $r$ is a continuous function of $\Delta$, this curve must cross $r=$ $c$ with $c \in(0,1)$ an odd number of times and with $c \in$ $(-\infty, 0) \cup(1, \infty)$ an even number of times. For $r>1$, the product of the two roots of the above quadratic is negative and hence does not have two positive roots. The case $r<0$ cannot arise because as mentioned earlier $\chi \in[0,1)$ and hence $\theta=\left(\frac{1-\rho^{A}}{1-\rho^{A} \chi}\right) \in\left[1-\rho^{A}, 1\right)$. Therefore $r$ decreases monotonically from 1 to 0 as $\Delta$ increases.

\subsection{Monotonicity of Number in System, $\rho^{H}>1$}

Complementary to Theorem 4, the following Theorem proves monotonicity of $E[N]$ for $\rho^{H}>1$ :

Theorem 5. When $\rho^{H}>1, E[N], E\left[N^{L}\right]$ and $E\left[N^{H}\right]$ increase monotonically as the switching rates decrease.

Proof. From (30)-(31), we can write the expectations of number of jobs in system as

$$
\begin{aligned}
E\left[N^{H}\right]= & {\left[\frac{\rho^{A}}{1-\rho^{A}}\right]+\left[\frac{\rho^{A}}{1-\rho^{A}}+\frac{\rho^{H}}{\rho^{H}-1}\right]\left[\frac{1-\ell_{\left(\rho^{H}>1\right)}}{-\ell_{\left(\rho^{H}<1\right)}^{\prime}}\right.} \\
& \left.\quad+\left(\frac{\ell_{\left(\rho^{H}>1\right)}^{\prime}}{-\ell_{\left(\rho^{H}<1\right)}^{\prime}}\right) r\right] \\
E\left[N^{L}\right]= & {\left[\frac{\rho^{A}}{1-\rho^{A}}\right]+\left[\frac{\rho^{A}}{1-\rho^{A}}-\frac{\rho^{L}}{1-\rho^{L}}\right]\left[\frac{1-\ell_{\left(\rho^{H}>1\right)}}{-\ell_{\left(\rho^{H}<1\right)}^{\prime}}\right.} \\
& \left.+\left(\frac{\ell_{\left(\rho^{H}>1\right)}^{\prime}}{-\ell_{\left(\rho^{H}<1\right)}^{\prime}}\right) r\right] \\
E[N]= & N_{\infty}+\left[N_{\infty}-N_{0}\right]\left(\frac{1-\ell_{\left(\rho^{H}>1\right)}}{-\ell_{\left(\rho^{H}<1\right)} \Delta}\right) \\
+ & {\left[N_{\infty}-N_{0}\right]\left(\frac{\ell_{\left(\rho^{H}>1\right)}}{\ell_{\left(\rho^{H}<1\right)}^{\prime}}\right) r }
\end{aligned}
$$

From the limits proved in (22)-(23), we know that for $\rho^{H}>$ $1, \lim _{\Delta \rightarrow \infty} r=0$ and $\lim _{\Delta \rightarrow 0} r=1$. We will now show that $r$ is monotonic in $\Delta$. This will imply that $E\left[N^{H}\right], E\left[N^{L}\right]$ and $E[N]$ all increase monotonically as switching rates decrease. Moreover $E[N]$ is bounded between two curves which are separated by the constant, $\mathcal{C}$,

$$
\mathcal{C}=\left[\frac{\rho^{A}}{1-\rho^{A}}+\frac{\frac{1}{\alpha^{H}} \frac{\rho^{H}}{\rho^{H}-1}-\frac{1}{\alpha^{L}} \frac{\rho^{L}}{1-\rho^{L}}}{\frac{1}{\alpha^{H}}+\frac{1}{\alpha_{L}}}\right]\left(\frac{\ell_{\left(\rho^{H}>1\right)}^{\prime}}{-\ell_{\left(\rho^{H}<1\right)}^{\prime}}\right)
$$

The proof of monotonicity of $r$ for this case will be along the same lines as the $\rho^{H}<1$ case. By substituting $\theta=$ $\ell_{\left(r h o^{H}>1\right)}-r \Delta \ell_{\left(\rho^{H}>1\right)}^{\prime}$ in (19), we get the following polynomial relating $\Delta$ and $r$ :

$$
\begin{aligned}
h(\Delta, r)= & r^{2}\left(\ell_{\left(\rho^{H}>1\right)}^{\prime}\right)^{2} \Delta^{2}\left[\left(\rho^{A}\right)^{2}+r \ell_{\left(\rho^{H}>1\right)}^{\prime}\left(\rho^{A}-\rho^{L}\right)\left(\rho^{H}-\rho^{A}\right)\right] \\
& -r \ell_{\left(\rho^{H}>1\right)}^{\prime} \Delta \rho^{A}\left[\frac{\rho^{A}\left(1-\rho^{A}\right)\left(\rho^{H}+\rho^{A}\right)}{\rho^{H}-\rho^{A}}\right. \\
& \left.+r \ell_{\left(\rho^{H}>1\right)}^{\prime}\left(\left(1-\rho^{A}\right)\left(\rho^{H}-\rho^{L}\right)-\left(\rho^{H}-1\right)\left(\rho^{A}-\rho^{L}\right)\right)\right] \\
& +\rho^{A} \rho^{H}\left(\frac{\rho^{A}\left(1-\rho^{A}\right)}{\rho^{H}-\rho^{A}}\right)^{2}(1-r)
\end{aligned}
$$

As before, the case $r<0$ cannot arise and for $r>1$ the product of the roots is negative. Following the same argument we have the desired results.

\section{RESULTS - SIMPLE APPROXIMATIONS}

In the previous section we showed that $E[N]$ is monotonic in $\alpha$. We now turn to the question of obtaining tight approximations for the $E[N]$ versus $\alpha$ curve. We seek approximations that are simple and can be easily analyzed. Evaluating these approximations yields insights into the behavior of $E[N]$ versus the system primitives, in particular $\alpha$.

We again consider the two cases: $\rho^{H}<1$ and $\rho^{H}>1$ separately. For each case, we first derive $r^{*}$, which is an approximation for the mixing ratio $r$ that fully specifies $E[N]$. This approximation $r^{*}$ is shown for the case $\rho^{H}<1$ in equation (42), and is shown for the case $\rho^{H}>1$ in equation (47). The $r^{*}$ approximation is highly accurate under all values of $\Delta$ (and hence $\alpha$ ), and yet it is a closed-form expression, which does not require the solution of a cubic.

We then go further by finding truly simple further approximations for $r^{*}$ in the case where $\Delta$ is "low" and the case where $\Delta$ is "high" (we make low and high precise in the coming sections). For the case $\rho^{H}<1, r_{\Delta: \text { high }}^{*}$ is shown in equation (44) and $r_{\Delta: \text { low }}^{*}$ is shown in equation (45). For the case $\rho^{H}>1, r_{\Delta: \text { :high }}^{*}$ is shown in equation (49) and $r_{\Delta: \text { low }}^{*}$ is shown in equation (50). Although these approximations $r_{\Delta: l o w}^{*}$ and $r_{\Delta: \text { high }}^{*}$ are intended to work only for "low" and "high" $\Delta$, we will find (see Figure 4) that using just these two expressions gives us an excellent sense of the shape of the $E[N]$ curve as a function of $\Delta$ (and hence $\alpha$ ).

\subsection{Approximations for $r$ when $\rho^{H}<1$}

As seen in the previous section (32)-(34), $r$ completely characterizes the behavior of $E[N]$ versus $\Delta$ (and hence $\alpha$ ). We now seek approximations for $r$ in terms of $\Delta$. We will state here without proof that $h(\Delta, r)$ from equation (35) is well approximated by the following polynomial in the region around the root of interest. Throughout, approximations will be denoted with a $*$ superscript.

$$
\begin{aligned}
\mathrm{h}^{*}(\Delta, r)= & r^{2}\left[\Delta^{2}\left(l_{\left(\rho^{H}<1\right)}^{\prime}\right)^{2}\left(1-\rho^{L}\right)\left(1-\rho^{H}\right)\right. \\
& \left.-\Delta l_{\left(\rho^{H}<1\right)}^{\prime}\left\{\left(\rho^{A}-\rho^{L}\right)\left(\rho^{H}-1\right)+\left(1-\rho^{L}\right)\left(\rho^{H}-\rho^{A}\right)\right\}\right] \\
& -r\left[\Delta l_{\left(\rho^{H}<1\right)}^{\prime}\left(1+\rho^{A}\right)\left(1-\rho^{L}\right)\left(1-\rho^{H}\right)\right. \\
& \left.+\rho^{A}\left(1-\rho^{L}\right)\left(1-\rho^{H}\right)\right] \\
& +\rho^{A}\left(1-\rho^{L}\right)\left(1-\rho^{H}\right)
\end{aligned}
$$

The expression $\mathrm{h}^{*}(\Delta, r)$ is obtained by ignoring the $r^{3}$ term in (35), and is a quadratic in both $\Delta$ and $r$. Simplify- 
ing (40) and making the following substitutions: $K=(1-$ $\left.\rho^{L}\right)\left(1-\rho^{H}\right)$ and $Z=\left\{\left(1-\rho^{L}\right)\left(\rho^{H}-\rho^{A}\right)-\left(1-\rho^{H}\right)\left(\rho^{A}-\rho^{L}\right)\right\}$, we get:

$$
\mathrm{h}^{*}(\Delta, r)=r^{2}\left[\Delta^{2} \rho^{A}-\Delta Z\right]-r\left[\Delta\left(1+\rho^{A}\right) K+K^{2}\right]+K^{2}
$$

From (41), we get a general approximation for $r$, denoted as $r^{*}$ :

$$
\begin{aligned}
r^{*} & =K \frac{\Delta\left(1+\rho^{A}\right)+K-\sqrt{\left(\Delta\left(1+\rho^{A}\right)+K\right)^{2}-4\left(\Delta^{2} \rho^{A}-\Delta Z\right)}}{2\left(\Delta^{2} \rho^{A}-\Delta Z\right)} \\
& =\frac{2\left(\frac{K}{\Delta}\right)}{\left(1+\rho^{A}\right)+\frac{K}{\Delta}+\sqrt{\left(1-\rho^{A}\right)^{2}+\left(\frac{K}{\Delta}\right)^{2}+2\left(\frac{\left(1+\rho^{A}\right) K+2 Z}{\Delta}\right)}}
\end{aligned}
$$

The sign before the discriminant in (42) has to be minus because

1. If the coefficient of $r^{2}$ is negative then the product of the roots is negative and minus sign will give the positive root.

2. If the coefficient of $r^{2}$ is positive then both roots are positive and minus sign will give the smaller of the roots.

We now look at the two different $\Delta$ regimes, low and high.

High $\Delta: \Delta \gg 2\left|\frac{\left(1+\rho^{A}\right) K+2 Z}{\left(1-\rho^{A}\right)^{2}}\right| \equiv u_{\left(\rho^{H}<1\right)}$

Starting from (43) and making appropriate approximations, we get,

$$
r_{\Delta: h i g h}^{*} \approx \frac{K}{\Delta+\frac{K+Z}{\left(1-\rho^{A}\right)}}
$$

Low $\Delta: \Delta \ll\left|\frac{K^{2}}{2\left(1+\rho^{A}\right) K+4 Z}\right| \equiv l_{\left(\rho^{H}<1\right)}$

Again, starting from (43) and making appropriate approximations:

$$
r_{\Delta: \text { low }}^{*} \approx \frac{K}{K+\frac{\left(K\left(1+\rho^{A}\right)+Z\right) \Delta}{K}}
$$

We defined the "high" $\Delta$ regime as $\Delta \gg u_{\left(\rho^{H}<1\right)}$ and the "low" $\Delta$ regime as $\Delta \ll l_{\left(\rho^{H}<1\right)}$. We will now provide very simple bounds on these thresholds. First observe that

$$
\left(1-\rho^{H}\right)\left(1+\rho^{L}\right)<\frac{\left(1+\rho^{A}\right) K+2 Z}{1-\rho^{A}}<\left(1-\rho^{L}\right)\left(1+\rho^{H}\right)
$$

Therefore,

$$
\begin{aligned}
u_{\left(\rho^{H}<1\right)} & =2\left|\frac{\left(1+\rho^{A}\right) K+2 Z}{\left(1-\rho^{A}\right)^{2}}\right| \\
& <2 \frac{\left(1-\rho^{L}\right)\left(1+\rho^{H}\right)}{\left(1-\rho^{A}\right)}<\frac{4}{\left(1-\rho^{A}\right)}
\end{aligned}
$$

and

$$
\begin{aligned}
l_{\left(\rho^{H}<1\right)} & =\left|\frac{K^{2}}{2\left(1+\rho^{A}\right) K+4 Z}\right| \\
& >\frac{\left(1-\rho^{L}\right)\left(1-\rho^{H}\right)^{2}}{2\left(1-\rho^{A}\right)\left(1+\rho^{H}\right)}>\frac{\left(1-\rho^{H}\right)^{2}}{2}
\end{aligned}
$$

Another salient question concerns the size of the area between the thresholds for the two regimes; how wide $\frac{u_{\left(\rho^{H}<1\right)}}{\left.l_{\left(\rho^{H}<1\right.}\right)}$ is:

$$
\begin{aligned}
\frac{u_{\left(\rho^{H}<1\right)}}{l_{\left(\rho^{H}<1\right)}} & =\left(2 \frac{\left(1+\rho^{A}\right) K+2 Z}{\left(1-\rho^{A}\right) K}\right)^{2} \\
& <\left(2 \frac{\left(1-\rho^{L}\right)\left(1+\rho^{H}\right)\left(1-\rho^{A}\right)}{\left(1-\rho^{A}\right)\left(1-\rho^{L}\right)\left(1-\rho^{H}\right)}\right)^{2} \\
& =\left(2 \frac{\left(1+\rho^{H}\right)}{\left(1-\rho^{H}\right)}\right)^{2} \\
& <\frac{16}{\left(1-\rho^{H}\right)^{2}}
\end{aligned}
$$

Therefore as $\rho^{H}$ increases to 1 , this gap increases. This defines the "transitional" regime for $\rho^{H}<1$.

\subsection{Approximations for $r$ when $\rho^{H}>1$}

Recall that for this case the combination parameter $r$ is the root of (39). We already know that for this case, the $E[N]$ curve is bounded between two curves separated by a constant. These two curves can be obtained by setting $r$ as 0 or 1 in (38). When $\rho^{H}$ is very close to 1 , then the gray area where neither of those curves is tight increases because the gap between these two curves also increases.

We will again state here that by neglecting $r^{3}$ term in (39), we get a tight approximation for $r$ that is quadratic. We will make the substitutions $K=\left(\rho^{H}-1\right)\left(\rho^{H}-\rho^{L}\right)$ and $Z=\left\{\left(1-\rho^{A}\right)\left(\rho^{H}-\rho^{L}\right)-\left(\rho^{H}-1\right)\left(\rho^{A}-\rho^{L}\right)\right\}$, yielding the approximation to $h(\Delta, r)$ as:

$\mathrm{h}^{*}(\Delta, r)=r^{2} \rho^{H}\left[\Delta^{2} \rho^{A}-\Delta Z\right]-r K\left[\Delta\left(\rho^{H}+\rho^{A}\right)+K\right]+K^{2}$

Following the same outline as Section 5.1,

$$
\begin{aligned}
r^{*} & =K \frac{\Delta\left(\rho^{H}+\rho^{A}\right)+K-\sqrt{\left(\Delta\left(\rho^{H}+\rho^{A}\right)+K\right)^{2}-4 \rho^{H}\left(\Delta^{2} \rho^{A}-\Delta Z\right)}}{2 \rho^{H}\left(\Delta^{2} \rho^{A}-\Delta Z\right)} \\
& =\frac{2\left(\frac{K}{\Delta}\right)}{\left(\rho^{H}+\rho^{A}\right)+\frac{K}{\Delta}+\sqrt{\left(\rho^{H}-\rho^{A}\right)^{2}+\left(\frac{K}{\Delta}\right)^{2}+2\left(\frac{\left(\rho^{H}+\rho^{A}\right) K+2 \rho^{H} Z}{\Delta}\right)}}
\end{aligned}
$$

High $\Delta: \Delta \gg 2\left|\frac{\left(\rho^{H}+\rho^{A}\right) K+2 \rho^{H} Z}{\left(\rho^{H}-\rho^{A}\right)^{2}}\right| \equiv u_{\left(\rho^{H}>1\right)}$ Starting from (48) and making approximations,

$$
r_{\Delta: h i g h}^{*}=\frac{K / \rho^{H}}{\Delta+\frac{K+Z}{\left(\rho^{H}-\rho^{A}\right)}}
$$

Low $\Delta: \Delta \ll\left|\frac{K^{2}}{2\left(\rho^{H}+\rho^{A}\right) K+4 \rho^{H} Z}\right| \equiv l_{\left(\rho^{H}>1\right)}$

$$
r^{*}{ }_{\text {low }} \approx \frac{K}{K+\frac{\left(K\left(\rho^{H}+\rho^{A}\right)+\rho^{H} Z\right) \Delta}{K}}
$$

By noting that

$$
\left(\rho^{H}-1\right)\left(\rho^{H}+\rho^{L}\right)<\frac{\left(\rho^{H}+\rho^{A}\right) K+2 \rho^{H} Z}{\rho^{H}-\rho^{A}}<\left(\rho^{H}-\rho^{L}\right)\left(\rho^{H}+1\right)
$$

we obtain the following bounds on thresholds $u_{\left(\rho^{H}>1\right)}$ and 
$l_{\left(\rho^{H}>1\right)}:$

$$
\begin{aligned}
u_{\left(\rho^{H}>1\right)} & =2\left|\frac{\left(\rho^{H}+\rho^{A}\right) K+2 \rho^{H} Z}{\left(\rho^{H}-\rho^{A}\right)^{2}}\right| \\
& <2 \frac{\left(\rho^{H}-\rho^{L}\right)\left(1+\rho^{H}\right)}{\left(\rho^{H}-\rho^{A}\right)}<\frac{2 \rho^{H}\left(\rho^{H}+1\right)}{\left(\rho^{H}-\rho^{A}\right)}
\end{aligned}
$$

and

$$
\begin{aligned}
l_{\left(\rho^{H}>1\right)} & =\left|\frac{K^{2}}{2\left(\rho^{H}+\rho^{A}\right) K+4 \rho^{H} Z}\right| \\
& >\frac{\left(\rho^{H}-\rho^{L}\right)\left(\rho^{H}-1\right)^{2}}{2\left(\rho^{H}-\rho^{A}\right)\left(1+\rho^{H}\right)}>\frac{\left(\rho^{H}-1\right)^{2}}{2\left(1+\rho^{H}\right)}
\end{aligned}
$$

Once again we seek information on how large the region between the two approximation regimes is:

$$
\begin{aligned}
\frac{u_{\left(\rho^{H}>1\right)}}{l_{\left(\rho^{H}>1\right)}} & =\left(2 \frac{\left(\rho^{H}+\rho^{A}\right) K+2 \rho^{H} Z}{\left(\rho^{H}-\rho^{A}\right) K}\right)^{2} \\
& <\left(2 \frac{\left(\rho^{H}-\rho^{L}\right)\left(1+\rho^{H}\right)\left(\rho^{H}-\rho^{A}\right)}{\left(\rho^{H}-\rho^{A}\right)\left(\rho^{H}-\rho^{L}\right)\left(\rho^{H}-1\right)}\right)^{2} \\
& =\left(2 \frac{\left(\rho^{H}+1\right)}{\left(\rho^{H}-1\right)}\right)^{2} \\
& <\frac{16}{\left(\rho^{H}-1\right)^{2}}
\end{aligned}
$$

Therefore as $\rho^{H}$ decreases to 1 , this gap increases.

\section{RESULTS - STOCHASTIC ORDERING}

Most results in queueing theory describe the experience of an arbitrary arrival to a system. But, in a time-varying system, an arrival may know that she is not "arbitrary"; she may know whether she is arriving into a high load or a low load period. In this case the salient question, as far as the arrival is concerned, is not about an arbitrary arrival's experience, but rather about her experience (conditional on the type of period into which she arrives).

To explore this question we compare, $N^{H}, N^{L}$, and $N^{\rho^{A}}$ stochastically, where the latter term denotes the number in system seen by an arrival to a stationary queue with the same average load, $\rho^{A}$, as our time-varying system.

Note that the distribution of future service rates, and thus response time, is completely determined by the number in system seen upon arrival and the type of period arrived into. Moreover, if only arrival rates vary (i.e. if service rates are constant), stochastic orderings for number in system immediately translate into stochastic orderings for response times.

Intuition leads one to believe that an arrival into a high load state should see more customers than one arriving into a low load state in expectation, but whether there is a stochastic dominance between these, that is, $N^{H} \geq_{s t} N^{L}$, is not obvious; we prove this to be true. Furthermore, one might also believe that an arrival during a high load state would see more customers than an arrival into the average system, and that an arrival into the average system would see more customer than an arrival during the low load state, $N^{H} \geq_{s t} N^{\rho^{A}} \geq_{s t} N^{L}$. Surprisingly, we find that this statement is only partially true: The first inequality holds but the second does not in general. Thus our system exhibits a striking lack of symmetry.

We start with a preliminary result:
Lemma 1. Given two copies of an $M / M / 1$ queue with load $\rho$, one starting with the queue's stationary distribution $\mathcal{X}$, and the other starting with an initial distribution $X(0)$, then

$$
\begin{gathered}
X(t) \geq_{s t} X(t+s) \geq_{s t} \mathcal{X} \quad \forall s, t \geq 0 \\
\Longleftrightarrow \\
\operatorname{Pr}\{X(0)=j\} \geq \rho \operatorname{Pr}\{X(0)=j-1\}
\end{gathered}
$$

The directions of all the inequalities can be reversed to get the condition for a stochastically increasing system.

Proof. Define the process $Y$ such that $Y(0)={ }_{s t} X(0)$. Set timers every $\exp (\lambda+\mu)$ units of time after which we toss a coin and have an arrival with probability $\frac{\mu}{\mu+\lambda}$ and a departure with probability $\frac{\lambda}{\mu+\lambda}$. Let the distribution just after the $i^{\text {th }}$ such event be $Y(i)$. A simple coupling argument shows that

$$
\begin{gathered}
X(t) \geq_{s t} X(t+s) \geq_{s t} \mathcal{X} \quad \forall s, t \geq 0 \\
\Longleftrightarrow \\
Y(i) \geq_{s t} Y(i+k) \geq_{s t} \mathcal{X} \quad \forall i, k \geq 0 .
\end{gathered}
$$

We first examine the condition for $Y(i+1) \leq_{s t} Y(i)$ :

$$
\begin{gathered}
\operatorname{Pr}\{Y(i+1) \geq j\} \leq \operatorname{Pr}\{Y(i) \geq j\} \\
\Longleftrightarrow \\
\operatorname{Pr}\{Y(i) \geq j-1\} \frac{\lambda}{\lambda+\mu}+\operatorname{Pr}\{Y(i) \geq j+1\} \frac{\mu}{\lambda+\mu} \leq \\
\operatorname{Pr}\{Y(i) \geq j\}\left(\frac{\lambda}{\lambda+\mu}+\frac{\mu}{\lambda+\mu}\right) \\
\Longleftrightarrow \\
\operatorname{Pr}\{Y(i) \geq j-1\}-\operatorname{Pr}\{Y(i) \geq j\}] \frac{\lambda}{\lambda+\mu} \leq \\
\operatorname{Pr}\{Y(i) \geq j\}-\operatorname{Pr}\{Y(i) \geq j+1\}] \frac{\mu}{\lambda+\mu} \\
\qquad \\
\operatorname{Pr}\{Y(i)=j-1\} \rho \leq \operatorname{Pr}\{Y(i)=j\}
\end{gathered}
$$

Thus $Y(i+1) \leq_{\text {st }} Y(i)$ iff $\operatorname{Pr}\{Y(i)=j\} \geq \rho \operatorname{Pr}\{Y(i)=$ $j-1\} \forall j \geq 1$, as required. Now, if $Y(i+1) \leq_{s t} Y(i) \forall i \geq 0$ it immediately follows that $Y(i) \geq_{s t} \mathcal{X}, \forall i \geq 0$, because $Y(n)$ converge in distribution to $\mathcal{X}$ as $n \rightarrow \infty$.

For $\rho \geq 1$, the system cannot decrease stochastically because the stationary distribution does not exist. The condition for such a system to be stochastic increasing is the same as that for an $M / M / 1$ with $\rho<1$.

THEOREM 6. For our alternating load system,

$$
N^{H} \geq_{s t} N^{L}
$$

Proof. By factoring the polynomials in the numerator and the denominator of $(10)$ we can write $\widehat{\Pi^{H}}(z)$ as:

$$
\begin{aligned}
\widehat{\Pi^{H}}(z) & =\frac{\lambda_{L} \mu_{H} \pi_{0}^{H}}{\lambda_{L} \lambda_{H}} \frac{(\delta-z)(z-\chi)}{(z-a)(z-b)(z-\chi)} \\
& =\frac{\pi_{0}^{H}}{\rho^{H}} \frac{(\delta-z)}{(z-a)(z-b)}
\end{aligned}
$$

where $0 \leq \chi<1$, with say $a \leq b$. (As mentioned in Section 3 , the denominator has a root $\chi$ in $[0,1)$ and the numerator must also have a root equal to $\chi$ for the $z$-transform to converge in the unit disc $|z|<1$.) Similarly

$$
\widehat{\Pi^{L}}(z)=\frac{\pi_{0}^{L}}{\rho^{L}} \frac{(\gamma-z)}{(z-a)(z-b)}
$$

The fact that $a, b, \chi, \delta$ and $\gamma$ are all real for $\rho^{A}<1$ can be easily verified. Also, $\delta, \gamma>a$ since the $z$-transform is 


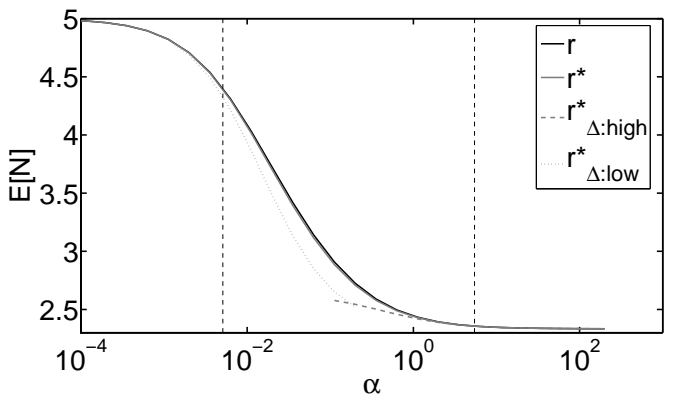

(a) $\rho^{H}=0.9, \rho^{L}=0.5$

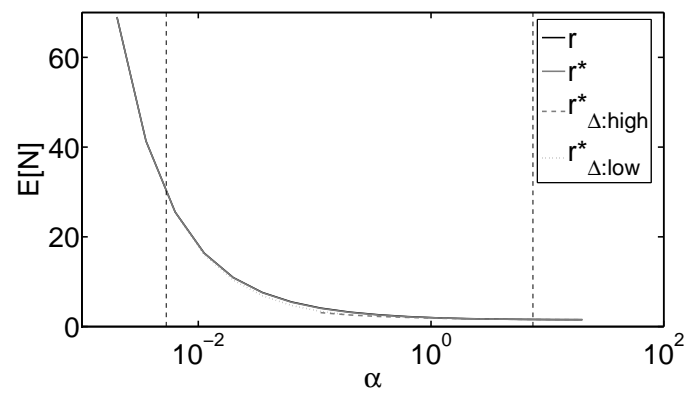

(c) $\rho^{H}=1.1, \rho^{L}=0.1$

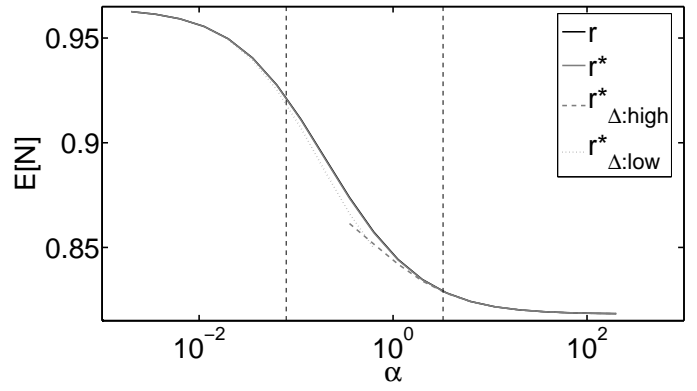

(b) $\rho^{H}=0.6, \rho^{L}=0.3$

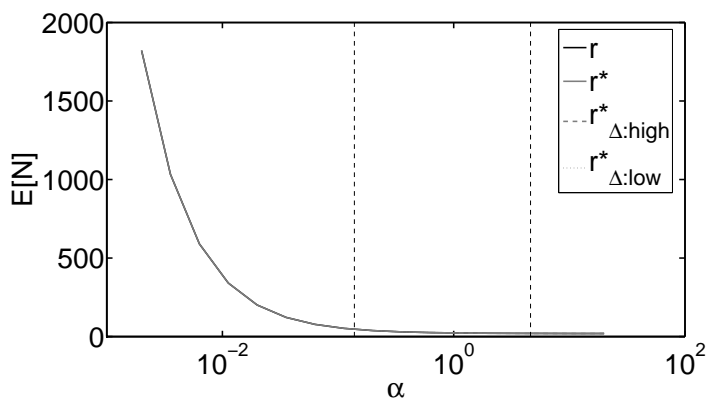

(d) $\rho^{H}=1.4, \rho^{L}=0.5$

Figure 4: Illustration of $\mathbf{E}[\mathbf{N}]$ as a function of $\alpha\left(=\alpha^{H}+\alpha^{L}\right)$, using the exact $r$; our closed-form approximation $r^{*}$; and our very simple approximations $r_{\Delta: l o w}^{*}$ and $r_{\Delta: h i g h}^{*}$. The top row shows examples where $\rho^{H}<1$. The bottom row shows examples where $\rho^{H}>1$. The vertical lines in each plot indicate the thresholds for the low $\Delta$ and high $\Delta$ regimes. In all cases $\mu^{H}=\mu^{L}=1$ and $\alpha^{H}=\alpha^{L}$.

an increasing function of $z$, it must become negative via a discontinuity. Using $\widehat{\Pi}^{L}(0)=\pi_{0}^{L}$ and $\widehat{\Pi^{H}}(0)=\pi_{0}^{H}$ :

$$
\delta=\rho^{H} a b, \quad \gamma=\rho^{L} a b
$$

Evaluating (14) for information about the roots $a$ and $b$, we have $f\left(1 / \rho^{H}\right) \geq 0, f(1)>0, f\left(1 / \rho^{A}\right) \leq 0, f\left(1 / \rho^{L}\right) \leq 0$. Therefore: $\max \left\{1, \frac{1}{\rho^{H}}\right\} \leq a \leq \frac{1}{\rho^{A}} \leq \frac{1}{\rho^{L}} \leq b$. Combining these with (53): $1<a \leq \gamma \leq b \leq \delta$.

Let $p_{i}^{H}=\operatorname{Pr}\left\{N^{H}=\bar{i}\right\}$; to derive $p_{i}^{H}$, we will expand (51).

$$
\begin{aligned}
\widehat{\Pi^{H}}(z) & =\frac{\pi_{0}^{H}(\delta-z)}{\rho^{H}(b-a)}\left[\frac{1}{a}\left(\frac{1}{1-z / a}\right)-\frac{1}{b}\left(\frac{1}{1-z / b}\right)\right] \\
& =\frac{\pi_{0}^{H}}{\rho^{H}(b-a)}\left[\left\{\left(\frac{\delta}{a}-1\right)-\left(\frac{\delta}{b}-1\right)\right\}\right. \\
& +z\left\{\left(\frac{\delta}{a}-1\right) \frac{1}{a}-\left(\frac{\delta}{b}-1\right) \frac{1}{b}\right\} \\
& \left.+z^{2}\left\{\left(\frac{\delta}{a}-1\right) \frac{1}{a^{2}}-\left(\frac{\delta}{b}-1\right) \frac{1}{b^{2}}\right\}+\cdots\right]
\end{aligned}
$$

Note that the last representation is what we would obtain by writing out the spectral expansion solution, with $\frac{1}{a}$ and $\frac{1}{b}$ as the two eigenvalues and the probability distribution as the sum of two geometric distributions.
Let $\nu_{i}^{H}=\frac{p_{i+1}^{H}}{p_{i}^{H}}$. From (54),

$$
\begin{aligned}
\nu_{i}^{H} & =\frac{\left(\frac{\delta}{a}-1\right) \frac{1}{a^{i+1}}-\left(\frac{\delta}{b}-1\right) \frac{1}{b^{i+1}}}{\left(\frac{\delta}{a}-1\right) \frac{1}{a^{i}}-\left(\frac{\delta}{b}-1\right) \frac{1}{b^{i}}} \\
& =\frac{\zeta u^{i+1}-\eta v^{i+1}}{\zeta u^{i}-\eta v^{i}} \\
& =\frac{\left(\zeta u^{i}-\eta v^{i}\right)\left(\zeta u^{i+2}-\eta v^{i+2}\right)}{\left(\zeta u^{i}-\eta v^{i}\right)\left(\zeta u^{i+1}-\eta v^{i+1}\right)}+\frac{\zeta \eta u^{i} v^{i}\left(u^{2}+v^{2}-2 u v\right)}{\left(\zeta u^{i}-\eta v^{i}\right)\left(\zeta u^{i+1}-\eta v^{i+1}\right)} \\
& =\nu_{i+1}^{H}+\frac{\zeta \eta u^{i} v^{i}(u-v)^{2}}{\left(\zeta u^{i}-\eta v^{i}\right)\left(\zeta u^{i+1}-\eta v^{i+1}\right)} \geq \nu_{i+1}^{H}
\end{aligned}
$$

Since the $p_{i}^{H}$ are a mixture of two geometrics, one decaying with rate $\frac{1}{a}$ and the other with $\frac{1}{b}$, and $\frac{1}{a} \geq \frac{1}{b}$, as $i$ increases the first component dominates and the rate of decay effectively becomes $\frac{1}{a}$; or, $\lim _{i \rightarrow \infty} \nu_{i}^{H}=\frac{1}{a} \geq \rho^{L}$. Also because $\nu_{i}^{H}$ are decreasing, $\nu_{i}^{H} \geq \frac{1}{a} \geq \rho^{L} \forall i$. Therefore starting an $M / M / 1$ with load $\rho^{L}$ and initial distribution as $N^{H}$ satisfies the conditions of Lemma 1 and will result in a stochastically decreasing process. $N^{L}$ is the random variable for the number of jobs at a time chosen from the distribution $\exp \left(\alpha^{L}\right)$, and is therefore also stochastically smaller than the initial distribution, $N^{H}$.

TheOREM 7. For our model: $N^{H} \geq_{s t} N^{\rho^{A}}$ but $N^{L} \nless_{s t} N^{\rho^{A}}$.

Proof. From the proof of Theorem $6, \nu_{i}^{H} \geq \nu_{i+1}^{H} \forall i \geq 0$ and $\lim _{i \rightarrow \infty} \nu_{i}^{H}=\frac{1}{a} \geq \rho^{A}$. Therefore, $\nu_{i}^{H} \geq \rho^{A} \forall i \geq 0$. Then using Lemma $1, N^{H} \geq_{s t} N^{\rho^{A}}$. 
Returning to the proof of Theorem 6 , define $q_{i}^{L}=\operatorname{Pr}\left\{N^{L} \geq\right.$ $i$. Using $\widehat{\Pi^{L}}(1)=1$ in (52),

$$
\frac{\pi_{0}^{L}}{\rho^{L}}=\frac{(a-1)(b-1)}{(\gamma-1)}
$$

Thus, using the formula for $p_{i}^{L}$, derived from the expansion of $\widehat{\Pi^{L}}(z)$ analogous to (54), and using (55):

$$
\begin{aligned}
q_{i}^{L} & =\sum_{j=i}^{\infty} p_{i}^{L} \\
& =\frac{\pi_{0}^{L}}{\rho^{L}(b-a)}\left[\left(\frac{\gamma}{a}-1\right) \frac{1}{a^{i}}\left(\frac{1}{1-\frac{1}{a}}\right)\right. \\
& \left.-\left(\frac{\gamma}{b}-1\right) \frac{1}{b^{i}}\left(\frac{1}{1-\frac{1}{b}}\right)\right] \\
& =\frac{(a-1)(b-1)}{(\gamma-1)(b-a)}\left[\left(\frac{\gamma-a}{a-1}\right) \frac{1}{a^{i}}+\left(\frac{b-\gamma}{b-1}\right) \frac{1}{b^{i}}\right]
\end{aligned}
$$

Let $c=\frac{(b-1)(\gamma-a)}{(\gamma-1)(b-a)}$. Then $q_{i}^{L}=c \frac{1}{a^{i}}+(1-c) \frac{1}{b^{i}}$ and $0 \leq c \leq 1$. Also recall that $a \leq \frac{1}{\rho^{A}}$. Let $k=\left\lceil\log _{a \rho^{A}} c\right\rceil+1$ so that $\left(a \rho^{A}\right)^{k}<c$. Now,

$$
q_{k}^{L}=c \frac{1}{a^{k}}+(1-c) \frac{1}{b^{k}} \geq \frac{c}{a^{k}}>\left(\rho^{A}\right)^{k}=q_{k}^{\rho^{A}}
$$

Clearly, $\forall j \geq k, q_{j}^{L}>q_{j}^{\rho^{A}}$ and hence $N^{L} \nless_{s t} N^{\rho^{A}}$. In fact, $N^{L}={ }_{s t} N^{\rho^{A}}$ if and only if $\rho^{A}=\rho^{H}=\rho^{L}$.

\section{CONCLUSION}

In this paper we have considered very basic, yet open, questions regarding the response time of a queue with timevarying load. We have found that the response time can both increase or decrease when the load fluctuates more slowly, and we have derived a simple slack criterion to specify the behavior. We have also proven the first monotonicity results for systems with time-varying load, as well as the first stochastic ordering results for these systems. Finally we have provided the first results on the shape of the mean response time in a queue with fluctuating load, as a function of the rate of fluctuation and other input primitives. These latter results were enabled by the derivation of a series of approximations for the mean number of jobs in the system, which are accurate and also very simple and closed-form, telling us exactly how the shape of the mean number of jobs is affected by the input primitives.

We hope that our research will stimulate others to ask further fundamental questions about time-varying systems. For example, we have seen that $E[N], E\left[N^{H}\right]$ and $E\left[N^{L}\right]$ are all monotonic in $\alpha$. We conjecture that a stronger result may exist, namely that the random variable $N^{H}$ is stochasically monotonic in $\alpha$. However this is entirely non-obvious, particularly since $N^{L}$ is not stochastically monotonic.

\section{REFERENCES}

[1] J. Abate, G. Choudhury, W. Whitt, Asymptotics for Steady-State Tail Probabilities in Structured Markov Queueing Models, Commun. Statist.-Stoch. Mod. 10(1), pp. 99-143, 1994.

[2] I. J. B. F. Adan, V. G. Kulkarni. Single-Server Queue with Markov-Dependent Inter-Arrival and Service Times, QUESTA 45, pp. 113-134, 2003.

[3] E. Arjas. On the Use of a Fundamental Identity in the Theory of Semi-Markov Queues, Adv. Appl. Prob. 4, pp. 271-284, 1972.
[4] S. Asmussen, J. Møller. Calculation of the Steady State Waiting Time Distribution in GI/PH/c and MAP/PH/c Queues, QUESTA 37, pp. 9-29, 2001.

[5] N. T. J. Bailey. A continuous time treatment of a simple queue using generating functions, J. R. Statist. Soc. B19, pp. 326-333, 1954.

[6] G. L. Choudhury, A. Mandelbaum, M. I. Reiman, W. Whitt. Fluid and Diffusion Limits for Queues in Slowly Changing Environments, Stoch. Mod. 13, pp. 121-146, 1997.

[7] E. Çinlar. Time Dependence of Queues with Semi-Markov Services, J. Appl. Prob. 4, pp. 356-364, 1967.

[8] E. Çinlar. Queues with Semi-Markov Arrivals, J. Appl. Prob. 4, pp. 365-379, 1967.

[9] A. B. Clark. A Waiting Line Process of the Markov Type, Ann. Math. Statist. 27, pp. 452-459, 1956.

[10] J. H. A. de Smit. The Single Server Semi-Markov Queue, Stoch. Proc. and Appl. 22, pp. 37-50, 1986.

[11] E. Gelenbe, C. Rosenberg. Queues with Slowly Varying Arrival and Service Processes, Man. Sci. 36(8), pp. 928-937, 1990.

[12] P. Harrison, H. Zatschler. Sojourn Time Distributions in Modulated G-Queues with Batch Processing, QEST 2004, Enschede, Netherlands, Los Alamitos, IEEE Computer Soc, pp. 90-99, 2004.

[13] D. P. Heyman On Ross's Conjectures about Queues with Non-Stationary Poisson Arrivals, J. Appl. Prob. 19, pp. 245-249, 1982.

[14] C. Knessl, Y. P. Yang. An Exact Solution for an $M(t) / M(t) / 1$ Queue with Time-Dependent Arrivals and Service, QUESTA 40, pp. 233-245, 2002.

[15] D. M. Lucantoni, K. S. Meier-Hellstern, M. Neuts. A Single Server Queue with Server Vacations and a Class of Non-Renewal Arrival Processes Adv. App. Prob. 22, pp. 676-705, 1990

[16] D. M. Lucantoni, M. Neuts. Some Steady-State Distributions for the MAP/SM/1 Queue, Commun. Statist.-Stoch. Mod. 10(3), pp. 575-598, 1994.

[17] W. A. Massey. Asymptotic Analysis of the Time Dependent $M / M / 1$ Queue, Math. of OR 10(2), pp. 305-327, 1985.

[18] I. Mitrani, R. Chakka. Spectral Expansion Solution for a Class of Markov Models: Application and Comparison with the Matrix-Geometric Method, Perf. Eval. 23(3), pp. 241-260, 1995.

[19] N. Miyoshi, T. Rolski. Ross Type Conjectures on Monotonicity of Queues, Festschrift for Daryl Daley, Phil Pollet and Peter Taylor Eds. Australian \& New Zealand J. of Stat. 46, pp. 121-132, 2004

[20] M. Neuts. The Single Server Queue with Poisson Input and Semi-Markov Service Times, J. Appl. Prob. 3, pp. 202-230, 1966.

[21] M. Neuts. The M/M/1 Queue with Randomly Varying Arrival and Service Rates, OPSEARCH 15(4), pp. 139-168, 1978.

[22] G. F. Newell. Queues with Time-Dependent Arrival Rates I The Transition Through Saturation, J. Appl. Prob 5, pp. 436-451, 1968

[23] G. F. Newell. Queues with Time-Dependent Arrival Rates II The Maximum Queue and the Return to Equilibrium, J. Appl. Prob 5, pp. 579-590, 1968.

[24] G. F. Newell. Queues with Time-Dependent Arrival Rates III A Mild Rush Hour, J. Appl. Prob 5, pp. 591-606, 1968.

[25] V. Ramaswami. The N/G/1 Queue and its Detailed Analysis, Adv. Appl. Prob. 12, pp. 222-261, 1980.

[26] K. L. Rider. A Simple Approximation to the Average Queue Size in the Time-Dependent M/M/1 Queue, JACM 23(2), pp. 361-367, 1976

[27] T. Rolski. Queues with Non-Stationary Input Stream: Ross's Conjecture, Adv. Appl. Prob. 13, pp. 603-618, 1981.

[28] S. Ross. Average Delay in Queues with Non-Stationary Poisson Arrivals, J. Appl. Prob. 15, pp. 602-609, 1978.

[29] B. Sengupta. A Queue with Service Interruptions in an Alternating Random Environment, OR 38(2), pp. 308-318, 1990.

[30] B. Sengupta. The Semi-Markov Queue: Theory and Applications, Commun. Statist.-Stoch. Mod. 6(3), pp. 383-413, 1990.

[31] T. Takine, Y. Matsumoto, T. Suda, T. Hasegawa. Mean Waiting Time sin Nonpreemptive Priority Queues with Markovian Arrival and i.i.d. Service Processes, Perf. Eval. 20, pp. 131-149, 1994.

[32] T. Takine, B. Sengupta. A Single Server Queue with Service Interruptions, QUESTA 26, pp. 285-300, 1997.

[33] Y. Yang, C. Knessl. Asymptotic Analysis of the M/G/1 Queue 
with Time-Dependent Arrival Rates, QUESTA 26, pp. 23-68, 1997.

[34] U. Yechiali, P. Naor. Queueing Problems with Heterogeneous Arrivals and Service, OR 19(3), pp. 722-734, 1971. 